\title{
Broadband SHF Direction-Finder
}

\author{
Sergey RADIONOV ${ }^{1}$, Igor IVANCHENKO ${ }^{1}$, Alexey KOROLEV ${ }^{2}$, Nina POPENKO ${ }^{1}$ \\ ${ }^{1}$ Usikov Inst. for Radiophysics and Electronics, National Academy of Sciences of Ukraine \\ 12 Ak. Proskura St., Kharkov, 61085, Ukraine \\ ${ }^{2}$ Inst. of Radioastronomy, National Academy of Sciences of Ukraine, 4 Krasnoznamennaya st., 61085, Kharkov, Ukraine \\ buran@ire.kharkov.ua
}

\begin{abstract}
The original design of the compact broadband direction-finder is presented in this paper. The cylindrical monopole antenna serves as a primary source of the reflector-type antenna. "Zero-amplitude" technique is used for bearing the SHF sources. The model experiments with the proposed direction-finder prototype in the frequency band $6 \mathrm{GHz}-11 \mathrm{GHz}$ have been carried out.
\end{abstract}

\section{Keywords}

Mobile communication, direction-finder, antenna measurement, antenna radiation pattern, "zero-amplitude" technique.

\section{Introduction}

Nowadays the radio systems operating at frequencies higher than $1 \mathrm{GHz}$ are widely used. The advancement to the state-of-the-art backbone links such as WiMAX standardsbased technology compels moving to the higher frequencies up to $66 \mathrm{GHz}$ that will allow one to deliver capacity of up to $40 \mathrm{Mbps}$ per channel, for fixed and portable access applications. With these remarks in mind, the source location problem should be overcome. The numerous approaches and techniques are known for detecting, identifying and classifying various types of disturbance sources [1]-[6]. Nevertheless, the compact broadband mobile direction-finders with high-rapid rates are required.

In this paper we present the reflector antenna for the compact X-band direction-finder and the results of model experiments.

\section{Design and Optimization of the Reflector Antenna}

Schematic view of the novel reflector antenna as the element of the direction-finder system is shown in Fig.1. This antenna consists of the main reflector and the primary source as the cylindrical monopole antenna located in the main reflector focus.

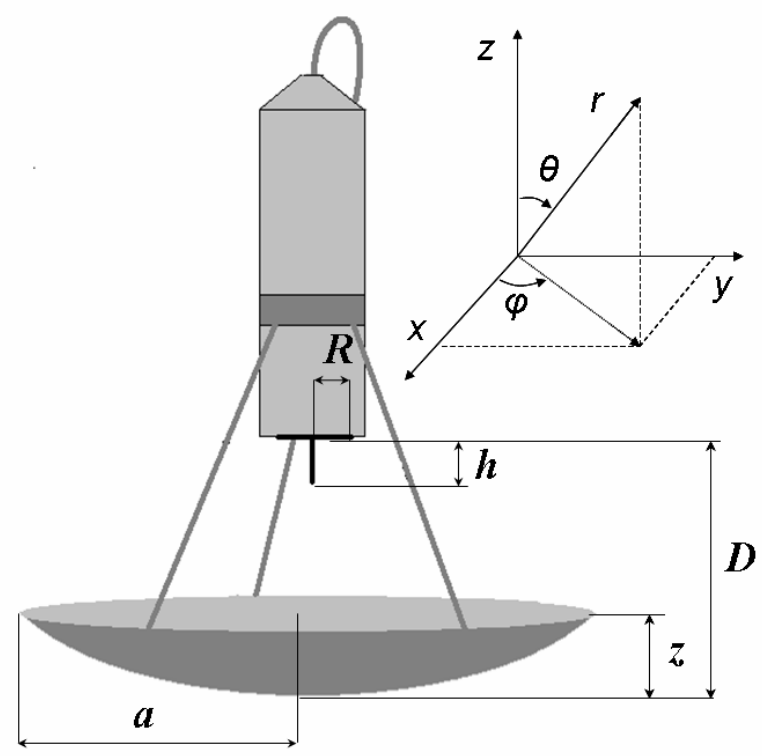

Fig. 1. Antenna design.

The monopole antenna is chosen from the following requirements, namely:

- the ground plane should have a minimal size to diminish the blockage effect;

- the EM field distribution on the reflector aperture should provide the maximal utilization factor of the reflector surface;

- the radiation pattern shape should remain virtually the same in the limits of the antenna bandwidth.

The investigations carried out by us earlier [7] have shown a possibility to control effectively the radiation characteristics of the cylindrical monopole antenna (Fig.2.) by changing the monopole height and the ground plane size. We have carried out the measurements of the monopole antenna characteristics when the geometrical parameters of antenna differed within the following limits: the monopole height $7.5 \mathrm{~mm}<h<22.5 \mathrm{~mm}$, and the ground plane radius $7.5 \mathrm{~mm}<R<22.5 \mathrm{~mm}$ with the step $7.5 \mathrm{~mm}$.

Since the primary source is located in the near-field of the main reflector, we focused on the investigations of near-field distributions in the radiating region of the mono 
pole antenna. The measurements were performed by means of the methods, facilities and special probe described in [8].

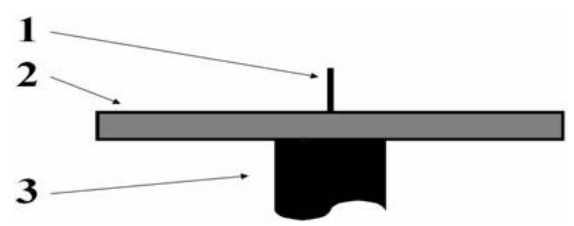

Fig. 2. Cylindrical monopole antenna: 1- monopole; 2- ground plane; 3- coaxial.

In the framework of these investigations some regularity in the near-field distributions have been determined for the fixed monopole height, namely: (i) the ground plane reduction results in the wave-beam narrowing and EM field power increasing outside the ground plane; (ii) the distance between the amplitude maxima in the near-field distribu tions increases with the ground plane radius increase. Such behavior of the EM field in the "radiating" region leads to the changes in the radiation patterns. For example, the near-field distributions and the radiation patterns of antenna with the monopole height $h=7.5 \mathrm{~mm}$ are shown in Figs. 3 and 4. Thick solid lines at the bottom of pictures in Fig. 3 show the ground plane size of the monopole antenna. As one sees from Fig. 4, there are different shapes of radiation patterns depending on the ground plane size. At that, with the ground plane radius increase the beamwidth increases, too. From the measurements of near-fields and radiation patterns we may conclude that the EM field distributions of the monopole antenna with parameters $h=7.5 \mathrm{~mm}, R=15 \mathrm{~mm}$ (Fig. $3 \mathrm{~b}$ and $4 \mathrm{~b}$ ) is the most acceptable one since, on one hand, the wave beam is wide enough for the effective illumination of the main reflector. On the other hand, the ground plane size is small from the feed blockage of the main reflector point of view.

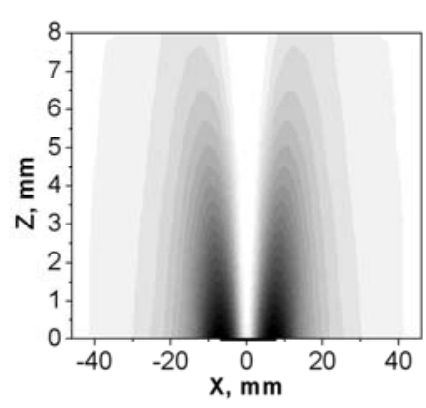

a

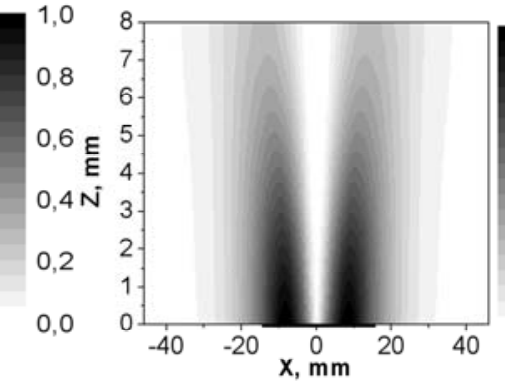

b

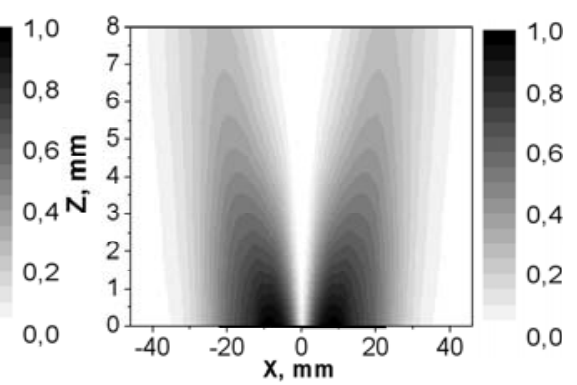

c

Fig. 3. Measured near-field distributions of the monopole antenna at the frequency $f=9.89 \mathrm{GHz}$ with different ground plane radii: $R=7.5 \mathrm{~mm}(\mathrm{a}) ; R=15 \mathrm{~mm}$ (b); $R=22.5 \mathrm{~mm}(\mathrm{c})$

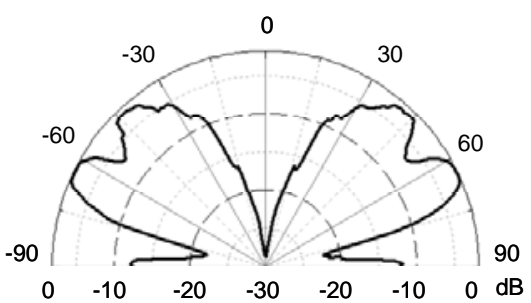

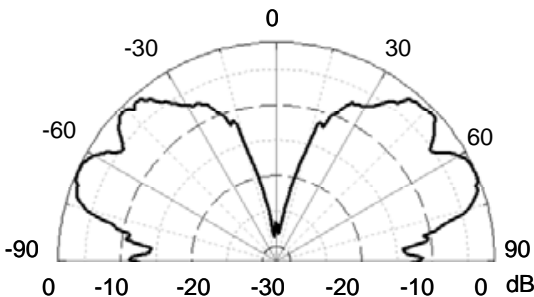

b

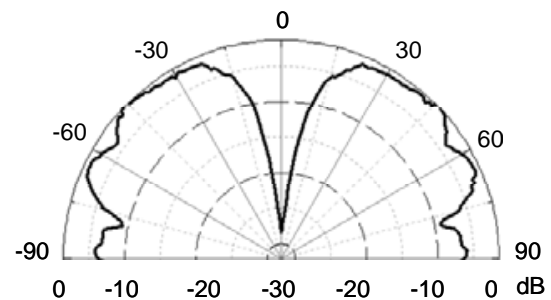

$\mathrm{c}$

Fig. 4. Measured radiation patterns of the monopole antenna at the frequency $f=9.89 \mathrm{GHz}$ with different ground plane radii: $R=7.5 \mathrm{~mm}$ (a); $R=15 \mathrm{~mm}$ (b); $R=22.5 \mathrm{~mm}$ (c)

Based on the experimental results noted above we have carried out the simulations of radiation patterns of the reflector antenna with a primary source as the monopole antenna with monopole height $h=7.5 \mathrm{~mm}, R=15 \mathrm{~mm}$, the focal length of the parabolic main reflector $D=55 \mathrm{~mm}$ and the aperture radius of the main reflector $a=80 \mathrm{~mm}$. The calculated radiation pattern of reflector antenna shows a high level of the side lobes (Tab. 1, Fig. 5a).

Therefore the computational modeling of the reflector antenna with parameters changed in the limits: $7.5 \mathrm{~mm}<h$
$<22.5 \mathrm{~mm}, 7.5 \mathrm{~mm}<R<22.5 \mathrm{~mm}, 50 \mathrm{~mm}<a<100 \mathrm{~mm}$, and $40 \mathrm{~mm}<D<60 \mathrm{~mm}$ was carried out. As a result of investigations noted above, the following optimal parameters of the reflector antenna were chosen, namely: the aperture radius of the main reflector is $a=80 \mathrm{~mm}$, the monopole height $h=22.5 \mathrm{~mm}$, the ground plane radius $R=22.5$ $\mathrm{mm}$, the focal length of the parabolic main reflector $D=55$ $\mathrm{mm}$. As can be seen from Fig. $5 \mathrm{~b}$ and Tab. 1, the calculated radiation pattern of the reflector antenna with optimal geometric parameters produces the radiation pattern with the low level of the first side lobe. 


\begin{tabular}{|c|c|c|c|c|}
\hline $\begin{array}{c}\mathrm{h}, \mathrm{R}, \\
(\mathrm{mm})\end{array}$ & $\begin{array}{c}\Delta \theta \\
(\text { degree })\end{array}$ & $\begin{array}{c}\Delta \theta^{\prime} \\
(\text { degree })\end{array}$ & $\begin{array}{c}\text { Side lobe } \\
\text { level (dB) }\end{array}$ & $\begin{array}{c}\text { Gain } \\
(\mathrm{dB})\end{array}$ \\
\hline \multicolumn{5}{|c|}{ Theory } \\
\hline $7.5,15$ & 9 & 1.5 & -15 & 16 \\
\hline $22.5,22.5$ & 10 & 1.5 & -21 & 18 \\
\hline \multicolumn{5}{|c|}{ Experiment } \\
\hline $22.5,22.5$ & 7 & 1.5 & -19 & 17 \\
\hline
\end{tabular}

Tab. 1. The characteristics of reflector antennas with different parameters.
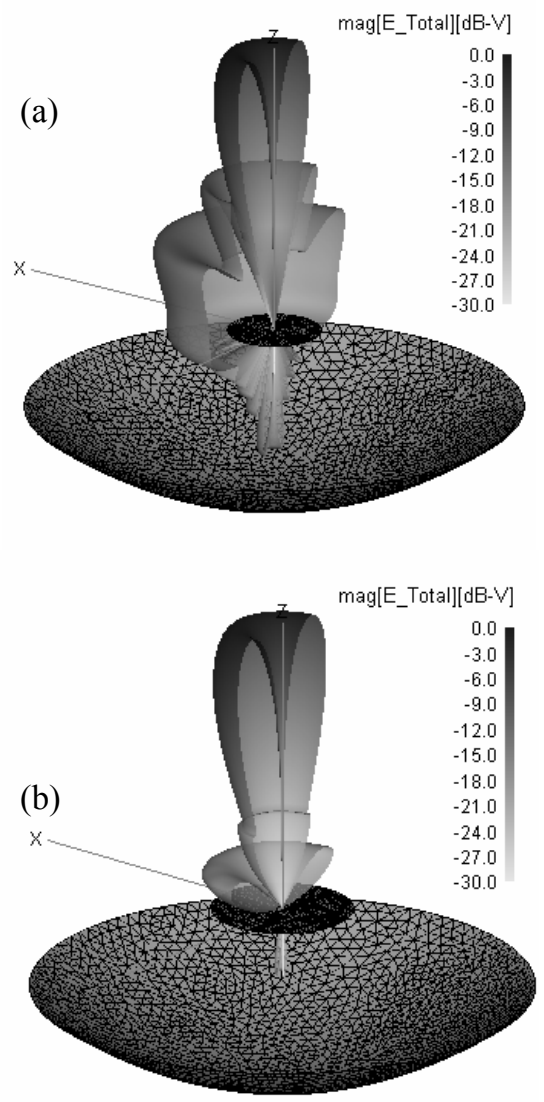

Fig. 5. Calculated radiation patterns of the reflector antennas with non optimal (a) and optimal (b) parameters.

The reflector antenna prototype with optimal geometrical parameters has been manufactured. The radiation patterns of reflector antenna prototype were measured in the sweep mode of the SHF oscillator [9] that allows one to analyze quickly the radiation pattern behavior in the limits of antenna bandwidth.

The measurements carried out in the far-field region have shown that the given reflector antenna produces the mono-beam conic radiation pattern (Fig. 6a) in the frequency band $6 \mathrm{GHz}-11 \mathrm{GHz}$ (Fig. 6b) with the elevation angle of peak directivity $\theta=10^{\circ}$. The experimental radiation pattern is narrower than the calculated one but the first side lobe level is higher (Tab. 1). It is worth noting that the power signal ratio in the antenna axis and in the main lobe maximum is less than $-20 \mathrm{~dB}$. At that the important characteristic of antenna from the point of view of its applying as a direction-finder antenna is the angle $\Delta \theta^{\prime}$. This angle determines the antenna position in which the signal increases on $3 \mathrm{~dB}$ in comparison with the minimal one. For the antenna prototype this angle is small that allows us to expect high space resolution of the proposed directionfinder.

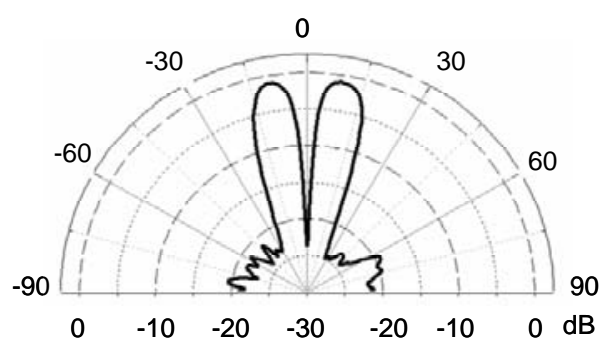

(a)

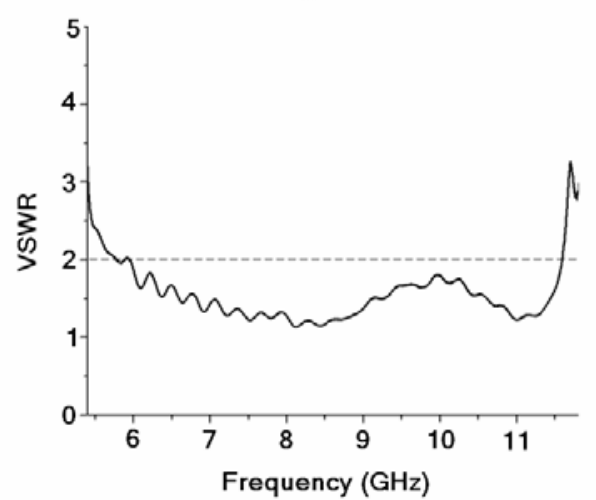

(b)

Fig. 6. Measured radiation pattern at $f=10 \mathrm{GHz}$ (a) and VSWR (b) of the direction-finder antenna prototype: $h=22.5$ $\mathrm{mm}, R=22.5 \mathrm{~mm}, D=55 \mathrm{~mm}, a=80 \mathrm{~mm}$.

With the aim to study the process of radiation shaping of the reflector antenna and to choose the optimal dimensions of the supporting antenna elements, as well as their mutual arrangement, we have carried out the simulations and measurements of EM field distributions in the radiating region of the reflector antenna prototype. The near-field distributions measured at the frequency $f=10 \mathrm{GHz}$ are shown in Fig.7a for the optimized direction-finder antenna prototype. As one sees from this picture one has the main lobe and the weak side lobe with a power level around $-15 \mathrm{~dB}$.

Computational modeling of the near-field distribution at the same frequency $f=10 \mathrm{GHz}$ (Fig. 7b) points out a qualitative agreement with the experiment. However, the calculated side lobe level is a little lower (around -20dB). Notice, that the power signal ratio in the antenna axis and in the main lobe maximum is higher than $-30 \mathrm{~dB}$. Thus, we can pronounce that the supporting elements do not disturb the EM field distribution in the radiating region of antenna prototype. 


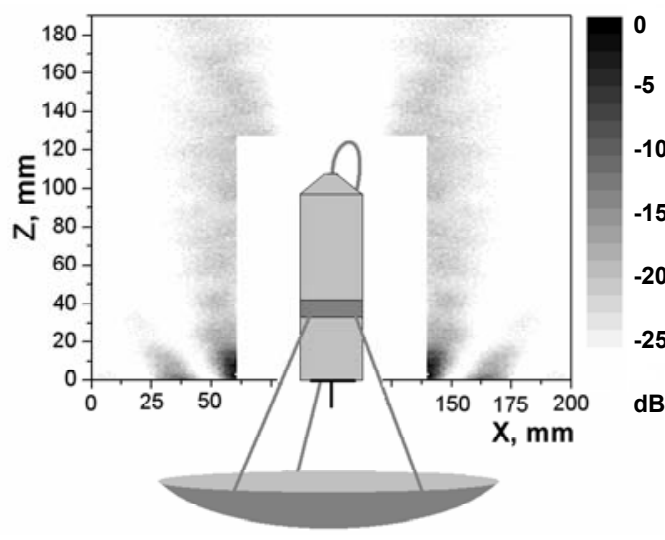

(a)

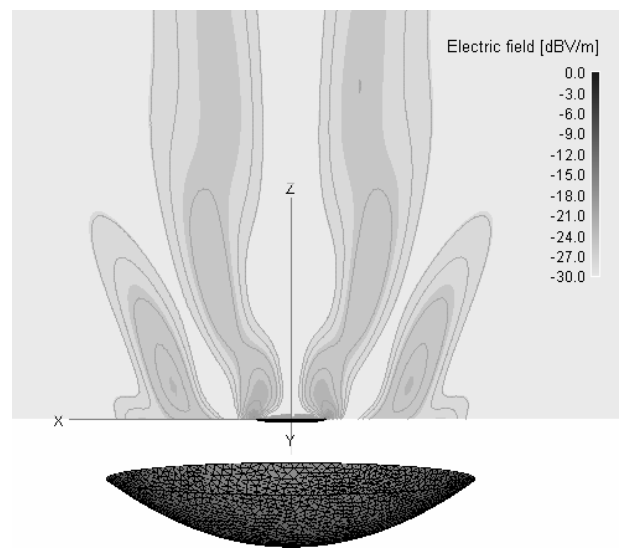

(b)

Fig. 7. Measured (a) and calculated (b) near-field distribution of the reflector-type antenna prototype.

\section{Direction-Finder Prototype}

In order to realize the reflector antenna operation in the received mode we have integrated the one-stage HEMT based amplifier in the input antenna circuit. The conceptual block-diagram of the direction-finder system is shown in Fig. 8. The signal received from the SHF source goes into the low-noise amplifier for the signal pre-amplification within the antenna bandwidth. Next logarithmic amplifier realizes the signal amplification to the level to be quite enough for the effective ultra wideband video detector operation $(U=1 \mathrm{mV})$. The wideband low-noise video amplifier with a small integration time constant $(\tau=0.1-$ 1 microseconds for the choice) increases the signal up to the level required to the stable flash encoder operation (around $2.5 \mathrm{~V}$ ). After video-amplifier the signal enters the interface and then on the working board of the control unit. The photo of the control unit is shown in Fig. 9.

There are two operating modes of the directionfinder, namely: (i) first, the rough determination of the source location on the maximum level of the received signal (so-called the "search mode"); (ii) second, the accurate determination of the source location on the global minimum of the received signal (so-called the "bearing mode"). In this case the arrangement of the light-emitting diodes on the working board of the control unit in the shape of $\mathrm{V}$-symbol simulates the aforementioned bearing algorithm (Fig. 10).

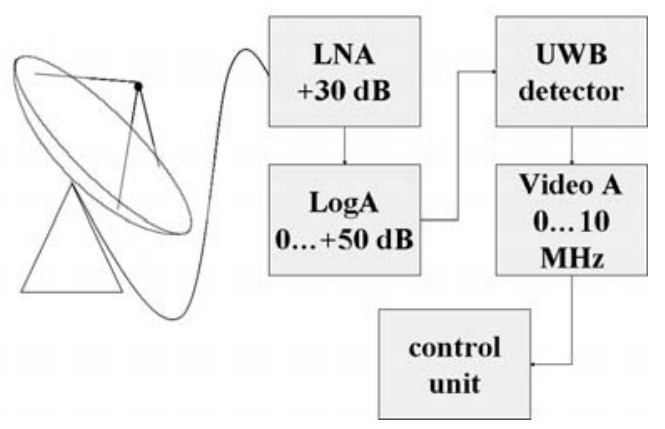

Fig. 8. Block-diagram of the direction finder system.

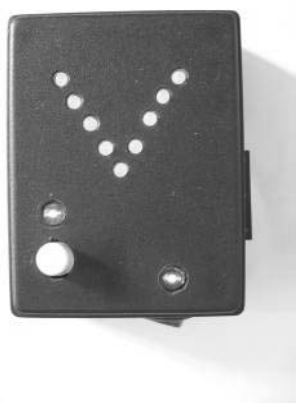

Fig. 9. Control unit.

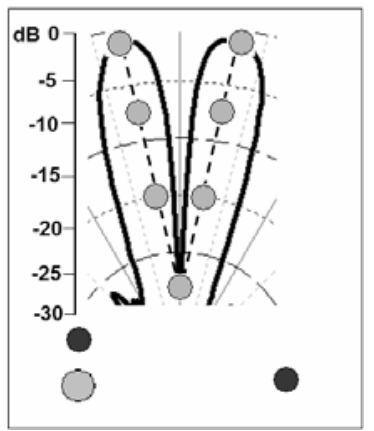

Fig. 10. Bearing angle imitator panel.

\section{Model Experiments}

We have carried out the model experiments on the SHF source bearing by means of the aforementioned direction-finder prototype. The open-ended X-band waveguide was located at the distance of $20 \mathrm{~m}$ from the directionfinder. We measured the angular dependences of the received signal power in the frequency band 6 to $11 \mathrm{GHz}$. Based on the analysis of experimental angular dependencies, which have been found to be similar to those depicted in Fig.10, we can pronounce that in the examined frequency band the power signal ratio in the antenna axis and in the main lobe maximum do not exceed $-20 \mathrm{~dB}$. The measurement results allow one to determine the accuracy of the source bearing approximate 2 degree. 


\section{Conclusions}

A novel design of the compact broadband directionfinder is presented. We have optimized the geometric parameters of the reflector antenna for its effective utilizing as a direction-finder antenna. The good matching of the reflector antenna to $50 \mathrm{Ohm}$ feeding line has been achieved in the antenna bandwidth $6 \mathrm{GHz}-11 \mathrm{GHz}$. The results of simulations and model experiments demonstrate the capability and benefits of the proposed direction-finder.

\section{References}

[1] ZOOGHBY, H., SOUTHALL, H. L., CHRISTODOULOU, C. G. Experimental validation of a neural network direction finder. In Proceedings of IEEE International Symposium on Antennas and Propagation, 1999, vol. 3, p. 1592-1595.

[2] PARSONS, A. C., GRADY, W. M., POWERS, E. J., SOWARD, J. C. A direction finder for power quality disturbances based upon disturbance power and energy. IEEE Transactions on Power Delivery, 2000, vol. 15 , no. 3, p. 1081-1086.

[3] CHAN, Y. T., LEE, B. H., INKOL, R., YUAN, Q. Direction finding with a four-element Adcock-Butler matrix antenna array. IEEE Transactions on Aerospace and Electronic Systems, 2001, vol. 37, no. 4, p. 1155-1162.

[4] KUWAHARA, Y., MATSUMOTO, T. Experiments on direction finder using RBF neural network with post-processing. Electronics Letters, 2005, vol. 41, no. 10, p. 602-603.

[5] MUELLER, R., LORCH, R., MENZEL, W. A. UHF direction finding antenna with optimized radar cross section. In Proceedings of IEEE International Symposium on Antennas and Propagation, 2006, p. $3255-3258$.

[6] KRAVETS, A., MORHART, C., BIEBL, E. Design of radio frequency direction finder for automotive locations. In Proceedings of $36^{\text {th }}$ European Microwave Conference, 2006, p. 714-717.

[7] IVANCHENKO, I. V., KHRUSLOV, M. M., KOROLEV, A. M., PAZYNIN, V. L., POPENKO, N. A. Effect of finite screen and monopole's height on radiation characteristics of monopole antenna. In Proceedings of $16^{\text {th }}$ International Conference on Microwaves, Radar and Wireless Communications MIKON 2006. Krakow (Poland), 2006, p. 729-731.

[8] IVANCHENKO, I. V., IVANCHENKO, D. I., KOROLEV, A. M., POPENKO, N. A. Experimental studies of X-band leaky-wave antenna performances. Microwave and Optical Technology Letters, 2002 , vol. 35 , no. 4 , p. $277-281$.

[9] ANDRENKO, A. S., IVANCHENKO, I. V., IVANCHENKO, D. I. KARELIN, S. Y., KOROLEV, A. M., LAZ'KO, E. P., POPENKO, N. A. Active broad X-band circular patch antenna. IEEE Antennas and Wireless Propagation Letters, 2006, vol. 5, p. 529-533.

\section{About Authors}

Sergey RADIONOV was born in Pervomayskiy, Ukraine, in 1984. In June 2007 he graduated from the Kharkov National University and received the MS degree in Radiophysics. From 2007 to the present he works as engineer at the Institute for Radiophysics \& Electronics of the National Academy of Sciences of Ukraine (IRE NASU). He has authored and co-authored 3 publications in the antenna field. S. Radionov is a Member of IEEE.

Alexey KOROLEV was born in Kharkov, Ukraine, in 1959. In June 1981 he graduated from the Kharkov State University and received the MS degree in Radiophysics. From 1981 to the present he works at the Institute for Radioastronomy of the National Academy of Sciences of Ukraine (IRA NASU). He received the Ph.D degree in Physics of devices, elements, and systems in 2005. From 2005 he is a Senior Researcher with the Department of Millimeter Waves of Radioastronomy in the IRA NASU. He has authored and co-authored more than 40 publications in the fields of low-noise microwave devices, high sensitive receivers and radiometers, micro - and millimeter wave antennas.

Igor IVANCHENKO was born in Kharkov, Ukraine, in 1952. In June 1975 he graduated from the Kharkov State University and received the MS degree in Radiophysics. From 1975 to the present he works at the Institute for Radiophysics \& Electronics of the National Academy of Sciences of Ukraine (IRE NASU). He received the Ph.D and D.Sc. degrees in Radiophysics in 1980 and 1997, respectively. From 1984 he is a Senior Researcher with the Department of Radio-Spectroscopy in the IRE NASU. He has authored and co-authored more than 100 publications in the fields of low-temperature radio-spectroscopy, semiconductor physics, antennas. He is currently the Head of the Laboratory of High Frequency Technology at IRE NASU. Prof. I. Ivanchenko is a Senior Member of IEEE.

Nina POPENKO was born in Gadyach, Ukraine, in 1948. In June 1971 she graduated from the Kharkov Institute of Radioelectronics and received the MS degree in Radiotechnik. From 1971 to the present he works at the Usikov Institute for Radiophysics and Electronics of the National Academy of Sciences of Ukraine (IRE NASU). He received the Ph.D and D.Sc. degrees in Radiophysics in 1981 and 1998, respectively. From 2007 she is a Leading Researcher with the Department of Radio-Spectroscopy in the IRE NASU. She has authored and co-authored more than 120 publications in the fields of low-temperature magnetic radio-spectroscopy, semiconductor physics, and antennas design. Prof. N. Popenko is a Senior Member of IEEE, and Member of EuMa. 